\title{
Schwangeren aktiv von Cannabis abraten
}

Fragestellung: Welche Risiken hat Cannabiskonsum in der Schwangerschaft?

Hintergrund: Marihuana wurde kürzlich in 29 US-amerikanischen Bundesstaaten sowie in Washington D. C. als Medikation unter anderem gegen Übelkeit zugelassen. Im Internet findet man Empfehlungen, Marihuana auch gegen Schwangerschaftsübelkeit einzusetzen. Cannabis ist in den USA die am häufigsten konsumierte Droge in der Schwangerschaft. Aus Daten der National Survey of Drug Use and Health von 2014 geht hervor, dass 3,85\% der Schwangeren zwischen 18 und 44 Jahren Cannabis konsumierten, 2012 waren es $2,37 \%$. THC (Tetrahydrocannabinol) als einer der Hauptwirkstoffe von Marihuana ist nur ein Partialagonist am CB1-Rezeptor. Synthetische Cannabinoide sind zumeist reine Agonisten am CB1-Rezeptor (und CB2-Rezeptor) und ihnen werden daher zum Teil andere und auch stärkere Effekte zugeschrieben. Jedoch ist der THC-Gehalt sowohl im medizinisch genutzten Marihuana als auch im missbrauchten Marihuana heutzutage deutlich angestiegen (1995 waren es im Schnitt 4\%, während es 2014 zirka 12\% waren).

Humanbefunde: Die Einflüsse von Cannabis auf die embryonale und fetale Entwicklung beim Menschen sind erst unzureichend untersucht. Allerdings ergaben Studien Hinweise auf

Volkow ND, Compton WM, Wargo EM. The risks of marijuana use during pregnancy. JAMA 2017; $317: 129-30$ ein erhöhtes Risiko für Anämie, niedriges Geburtsgewicht und die Notwendigkeit einer intensivmedizinischen Überwachung bei Neugebo- renen, deren Mütter in der Schwangerschaft Cannabis konsumierten. Zudem wurde eine Beeinträchtigung höherer Exekutivfunktionen (wie Impulskontrolle, visuelles Gedächtnis, Aufmerksamkeit) bei pränatal exponierten Schulkindern gefunden.

Tiermodell: Aus Studien mit synthetischen Cannabinoiden, die noch stärker mit den Cannabinoidrezeptoren interagieren wie beschrieben, weiß man, dass diese teratogene Effekte im Tierversuch haben. Bei Mäusen wurden nach Cannabinoidexposition (synthetisches Cannabinoid CP-55.940) im Mutterleib Gehirn-, Augen- und Gesichtsfehlbildungen gefunden. Die Anzahl an Mäusen mit Fehlbildungen korrelierte linear mit der Dosis: je höher diese war, desto mehr fehlgebildete Mäuse. Demgegenüber gibt es bei Menschen noch keine Hinweise auf ähnliche Effekte. Synthetische Cannabinoide sind aber auch erst seit wenigen Jahren in Gebrauch.

Schlussfolgerungen: Die Autoren fordern weitere Untersuchungen, insbesondere mit hochdosierten THC-Präparationen und synthetischen Cannabinoiden, um den Effekt auf die neuronale Entwicklung bei pränataler Exposition abschätzen zu können. Ein Problem dabei ist auch die Trennung des Effekts von Cannabis/Cannabinoiden von Alkohol, Tabak und anderen (illegalen) Substanzen, die in dieser Personengruppe ebenfalls häufig konsumiert werden. Auch haben Frauen, die in der Schwangerschaft Drogen konsumieren, weitere, potenziell für den Fetus schädigende Verhaltensweisen. Die Autoren kommen zu dem Schluss, dass Schwangeren aktiv vom Cannabiskonsum abgeraten werden sollte, auch nicht zur Behandlung von Hyperemesis gravidarum.

\section{- Kommentar von Sarah Kittel-Schneider, Frankfurt am Main}

\section{Gegen Schwangerschaftsübelkeit gibt es bessere Alternativen}

In dieser Stellungnahme wird die Datenlage bezüglich Vorkommen und Risiken von Cannabis- und Cannabinoidkonsum in der Schwangerschaft diskutiert. Hintergrund ist die Legalisierung von Marihuana in medizinischer Anwendung in über der Hälfte der US-amerikanischen Bundesstaaten. Auch in kleineren, neueren Studien konnte man in Umfragen von schwangeren Frauen sehen, dass diese auch in der Schwangerschaft Cannabis konsumieren. Und 10\% dieser Frauen gaben an, dass sie auch mehr konsumieren würden, wenn es legal wäre [1]. Da auch in Deutschland demnächst Cannabis als Medikament auf Rezept erhältlich sein soll, sind weitere Studien wichtig, um das Risiko für das ungeborene Kind bei pränatalem Konsum besser abschätzen zu können. Bis dahin sollte aufgrund der existierenden Datenlage Frauen mit Kinderwunsch und Schwangeren vom Konsum von Cannabis- und insbesondere Cannabinoidprodukten dringend abgeraten werden. Cannbinoide wirken als volle CB1- und CB2-Agonisten und haben daher vermutlich schädlichere Wirkungen als THC [2]. Gegen Schwangerschafts- übelkeit gibt es weit besser untersuchte Alternativen, wie zum Beispiel Dimenhydrinat oder Mirtazapin [3].
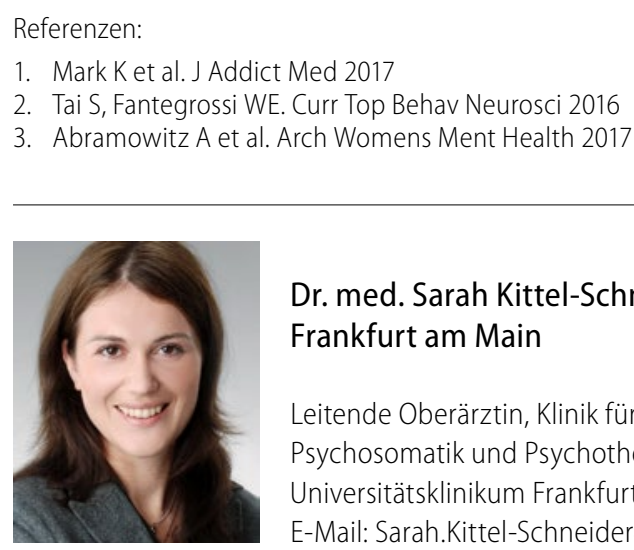

Dr. med. Sarah Kittel-Schneider, Frankfurt am Main

Leitende Oberärztin, Klinik für Psychiatrie,

Psychosomatik und Psychotherapie,

Universitätsklinikum Frankfurt am Main

E-Mail: Sarah.Kittel-Schneider@kgu.de 